\title{
Lifting the Veil: The Quest for Noninvasive Biomarkers for the Accurate Diagnosis of Eosinophilic Esophagitis
}

\author{
Alex Straumann ${ }^{1} \cdot$ Thomas Greuter $^{1}$ \\ Published online: 10 July 2020 \\ (c) Springer Science+Business Media, LLC, part of Springer Nature 2020
}

Eosinophilic esophagitis (EoE) is a hidden disease: Firstly, its concealed localization deep within the chest cavity confounds access; secondly, solid food dysphagia, the leading symptom of EoE, is often masked by adapted eating habits such as food modification and avoiding strategies, and finally, EoE is a chronic inflammatory disease with only minimal systemic manifestations.

Though EoE is a chronic inflammatory disorder with a noticeably increasing prevalence over the last two decades [1], its definition and diagnostic criteria have not changed much over the time span. Since its first description in this journal by Stephen Attwood more than 25 years ago, this condition has been primarily defined clinicopathologically [2]. Clinically, the definition of EoE almost exclusively relies on subjective symptoms (patient-reported outcomes), as physical examination and laboratory analyses are often unremarkable [3]. Pathologically—in contrast-EoE is based on objective biological criteria, namely an eosinophil-predominant inflammation of the esophageal squamous epithelium [3]. Since symptoms and inflammatory activity are only modestly correlated [4], diagnosis and disease monitoring require a careful history and an upper endoscopy with structured tissue sampling and subsequent pathological examination of the specimens [3]. These procedures are time-consuming, and the latter is in addition invasive and cost-intensive. A less- or even noninvasive method to determine inflammatory activity is therefore a major unmet need for diagnosing and monitoring of EoE.

As of yet, almost all attempts to find a single marker in serum, blood, saliva or feces reliably mirroring esophageal eosinophilia have failed [5]. Nevertheless, flow cytometric determination of eosinophil progenitors in the peripheral

Alex Straumann

alex.straumann@hin.ch

1 Department of Gastroenterology and Hepatology,

University Hospital Zurich, Raemistrasse 100, 8091 Zurich,

Switzerland blood is a promising method according to preliminary data $[6,7]$. Less invasive tools such as the esophageal string or sponge test $[8,9]$ are potential options, but they are neither approved nor readily available.

In this Issue of Digestive Disease and Sciences, Lingblom et al., a Swedish group of EoE experts, present an interesting and promising new approach to address this relevant research gap [10]. The authors measured a panel of bloodbased biomarkers obtained in 20 adult patients with active EoE with severe symptoms, comparing them with inflammatory activity in the esophageal tissue. After 2 months of treatment with swallowed topical corticosteroids, assessments were repeated. Using multivariate analyses, a combination of 13 blood biomarkers and 10 symptom components facilitated the separation of treatment responders from nonresponders with an acceptable power. Unfortunately, blood biomarkers alone were not sufficient to unequivocally separate the two groups.

The readout instrument used in this study is complex: firstly because it was a combination of symptoms and biomarkers, and secondly because a whole panel and not a single biomarker was applied. Despite its potential, it effectively illustrates how difficult it is to reliably determine the inflammatory activity of EoE by noninvasive measures. The dilemma is that single biomarkers in EoE are likely an unrealistic goal and combination approaches are probably too complex to be applied toward daily clinical practice.

To summarize, EoE still is a hidden disease, and determining its activity in children and adults remains a challenge. The availability of meaningful and reliable noninvasive biomarkers is key in clinical practice as well as for research. The study by Lingblom and colleagues is another important piece in the complex puzzle. Further studies are urgently needed, either through simplifying the instrument used in this study and making it more sensitive, or by developing alternative methods. 


\section{References}

1. Straumann A, Katzka DA. Diagnosis and treatment of eosinophilic esophagitis. Gastroenterology. 2018;154:346-359.

2. Attwood SE, Smyrk TC, Demeester TR, et al. Esophageal eosinophilia with dysphagia, a distinct clinicopathologic syndrome. Dig Dis Sci. 1993;38:109-116. https://doi.org/10.1007/BF01296781.

3. Furuta GT, Katzka DA. Eosinophilic esophagitis. $N$ Engl J Med. 2015;373:1640-1648.

4. Safroneeva E, Straumann A, Coslovsky M, et al. Symptoms have modest accuracy in detecting endoscopic and histologic remission in adults with eosinophilic esophagitis. Gastroenterology. 2016;150:581-590.

5. Schlag C, Miehlke S, Heiseke A, et al. Peripheral blood eosinophils and other non-invasive biomarkers can monitor treatment response in eosinophilic oesophagitis. Aliment Pharmacol Ther. 2015;42:1122-1130.

6. Morris DW, Stucke EM, Martin LJ, et al. Eosinophil progenitor levels are increased in patients with active pediatric eosinophilic esophagitis. J Allergy Clin Immunol. 2016;138:915-918.
7. Henderson A, Magier A, Schwartz JT, et al. Monitoring eosinophilic esophagitis disease activity with blood eosinophil progenitor levels. J Ped Gastro Nutr. 2020. (publish ahead of print).

8. Furuta GT, Kagalwalla AF, Lee JJ, et al. The oesophageal string test: a novel, minimally invasive method measures mucosal inflammation in eosinophilic oesophagitis. Gut. 2013;62:1395-1405.

9. Katzka DA, Geno DM, Ravi A, et al. Accuracy, safety, and tolerability of tissue collection by cytosponge vs endoscopy for evaluation of eosinophilic esophagitis. Clin Gastroenterol Hepatol. 2015;13:77-83.

10. Lingblom C, Albinsson S, Johansson L, et al. Patient-reported outcomes and blood-based parameters identify response to treatment in eosinophilic esophagitis. Dig Dis Sci. (Epub ahead of print). https://doi.org/10.1007/s10620-020-06368-2.

Publisher's Note Springer Nature remains neutral with regard to jurisdictional claims in published maps and institutional affiliations. 